\title{
Aneurisma verdadeiro bilateral de artéria tibial posterior
}

\author{
True bilateral aneurysm of the posterior tibial artery
}

\section{Silvio Romero de Barros M arques', Rodrigo Augusto de Aguiar G uedes², Esdras M arques Lins ${ }^{2}$, Belchior M edeiros de Lucena ${ }^{3}$, C arlos Alexandre Albuquerque M aranhão3, Emmanulle Tenório3}

\section{Resumo}

N este artigo, faz-se o relato de um caso de aneurisma verdadeiro bilateral da artéria tibial posterior em paciente de 57 anos. $0 \mathrm{~s}$ aneurismas surgiram em épocas diferentes. 0 s aspectos clínicos, diagnósticos e terapêuticos deste caso são discutidos. Este relato é importante, pois os autores não têm conhecimento de caso semelhante na literatura consultada.

Palavras-chave: Aneurisma arterial periférico, aneurisma arterial verdadeiro dos membros inferiores, aneurisma da artéria tibial posterior.

Os aneurismas arteriais periféricos verdadeiros das extremidad esinferiores são menos comuns que os aneurismas da aorta abdominal, mas os relatos sobre o seu diagnóstico e tratamento são anteriores aos dos aneurismas aórticos, tal vez devido à maior acessibilidade aos mesmos ${ }^{1-3}$.

1. Doutor, Professor adjunto, Disciplina de Cirurgia Vascular, Centro de Ciências da Saúde, Universidade Federal de Pernambuco (CCS-UFPE), Recife, PE.

2. Professor substituto, Disciplina de Cirurgia Vascular, CCS-UFPE, Recife, PE.

3. Residente, Serviço de Cirurgia Vascular, Hospital de Clínicas da Universidade Federal de Pernambuco (HC-UFPE), Recife, PE.

O presente estudo foi realizado no HC-UFPE, Recife, PE, e apresentado como pôster no XXXVI Congresso Brasileiro de Angiologia e Cirurgia Vascular.

Artigo submetido em 12.01.06, aceito em 20.03.06.

Copyright $\odot 2006$ by Sociedade Brasileira de Angiologia e de Cirurgia Vascular.

\begin{abstract}
This article reports a case of true bilateral aneurysm of the tibial posterior artery in a 57 year-old patient. The aneurysms occurred at different times. The clinical, diagnostic and therapeutic aspects of this case are discussed. This report is important because the authors did not find a similar description in the literature.
\end{abstract}

Key words: Peripheral arterial aneurysm, true arterial aneurysm of lower limbs, aneurysm of the tibial posterior artery.

N os membros inferiores, a artéria poplítea éa mais freqüentemente acometida, e comumente estes aneurismas estão associados ao aneurisma da aorta abdominal, principalmentequando bilateral. A artériafemoral éo segundo sítio mais comum dos aneurismas arteriais periféricos verdadeiros, enquanto que os aneurismas das artérias tibiais são raros ${ }^{4-11}$.

O s aneurismas arteriais periféricos verdadei ros são causados principalmente por aterosclerose, e o seu diagnóstico é estabelecido através da avaliação clínica, onde é possível detectar tumor pulsátil no trajeto arterial. A confirmação diagnóstica é geralmente feita através da avaliação com métodos de imagem, como 0 eco-D oppler, a angiorressonância e a arteriografia. D e forma diferente dos aneurismas da aorta abdominal, os aneurismas arteriais periféricos raramente apresentam ruptura, sendo a trombose e a embolia suas principais complicações ${ }^{1,2,7-9,12}$.

0 objetivo deste relato é descrever os aspectos etiológicos, o diagnóstico clínico, os exames complementares e o tratamento envolvidos neste caso. 


\section{Relato do caso}

Paciente do sexo masculino, 57 anos, pardo, carpinteiro, natural e procedente do Recife $(P E)$, sem comorbidades ou antecedentes patológicos e com exames bioquímicos, incluindo as provas inflamatórias, normais.

Inicialmente, foi admitido no Serviço de Cirurgia Vascular do Hospital das Clínicas da Universidade Federal de Pernambuco, em janeiro de 2003, com a história de surgimento, há 6 meses, de tumoração pulsátil dolorosa e expansiva em face medial do tornozelo direito. Ao exame físico, foi observado que a tumoração apresentava aproximadamente $3,5 \times 2,0 \mathrm{~cm}$ no seu maior diâmetro. 0 paciente não apresentava história detrauma, infecçõese/ ou punções real izadasna topografia da lesão.

A hipótese diagnóstica ao exame clínico era de aneurisma da artéria tibial posterior (ATP) direita, que foi confirmado pelo eco-D oppler colorido arterial realizado em 17 de dezembro de 2002 e apresentado pelo paciente no momento do internamento. 0 laudo do eco-D oppler evidenciava a presença de dilatação aneurismática da ATP direita, que apresentava diâmetro máximo de 3,0 ×2,2 cm, sem trombo no seu interior e com fluxos amplos e trifásicos em todas as artérias da pernadireita. N o membro inferior esquerdo, foi encontrada imagem heterogênea, bem delimitada, medindo em torno de $6,5 \times 4,3 \mathrm{~cm}$ no terço médio da perna e projetando-se na sua face interna.

Posteriormente, foi realizada uma arteriografia dos membrosinferiores, que evidenciou aneurisma fusiformeda ATP direita (Figura 1) e ausência de visualização da artéria fibular esquerda (Figura 2), que apresentava imagem compatível com aneurisma trombosado em seu segmento distal; as demais artérias dos membros inferiores eram normais.

Em 21 dejaneiro de2003, o pacientefoi submetido a tratamento cirúrgico, sendo realizada aneurismectomia com ligadura dos cotos proximal e distal da ATP direita, recebendo alta no terceiro dia pós-operatório (D PO) sem intercorrências.

O histopatológico realizado foi compatível com aneurisma verdadeiro de ATP direita.

Por motivos sociais, retornou ao serviço apenas em dezembro de 2004, com história de surgimento de tumoração pulsátil e dolorosa, há 4 meses, na região maleolar medial esquerda. Segundo o relato do paciente, a tumoração apresentou crescimento rápido, não havendo história de trauma ou infecção. A repetição das provas inflamatórias não demonstrou anormalidades.

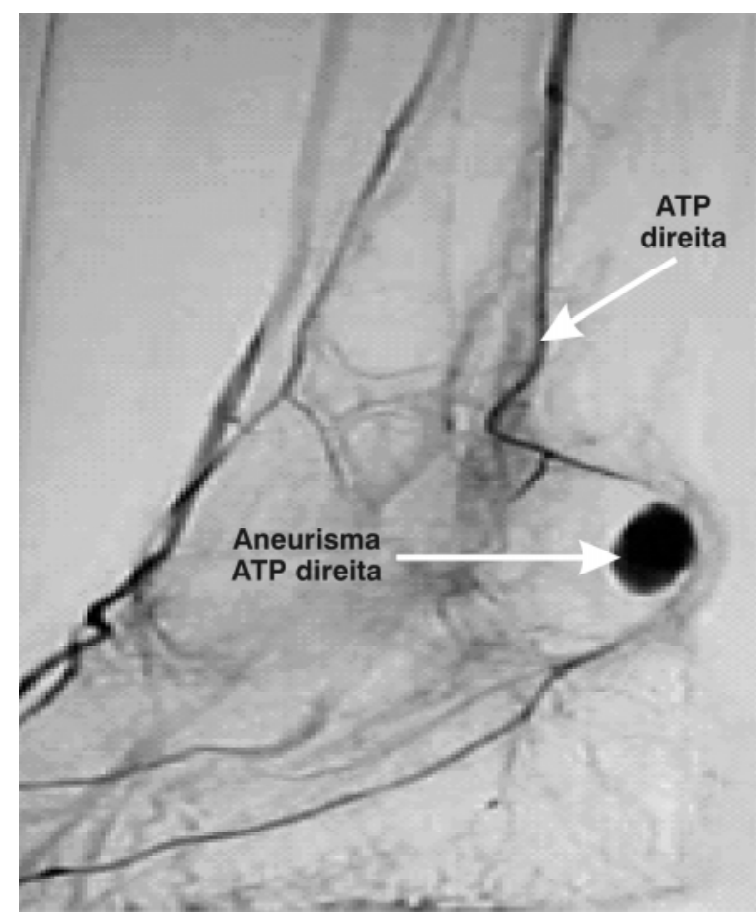

Figura 1 - Arteriografia de membro inferior direito, realizada no primeiro internamento, demonstrando aneurisma fusiformedaAT P ATP $=$ artéria tibial posterior.

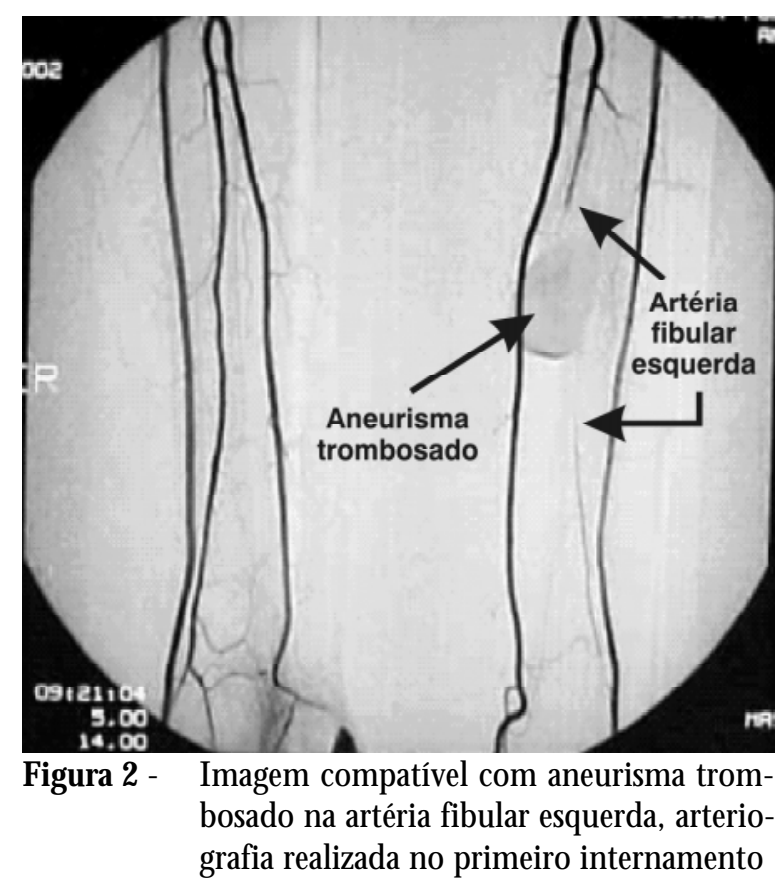


Realizou nova arteriografia, que evidenciou aneurisma fusiformedeATP esquerda (Figura 3), eo restante do exame era normal, exceto pela não-visualização distal da artéria peroneira, já descrita na arteriografia anterior.

Em 4 dejaneiro de 2004, o paciente foi submetido a tratamento cirúrgico, sendo realizadas aneurismectomia einterposição de enxerto venoso para manutenção da perviedade arterial. 0 pacienterecebeu al ta hospitalar no quarto D PO sem intercorrências.

0 estudo histopatológico realizado foi compatível com aneurisma verdadeiro de AT P esquerda.

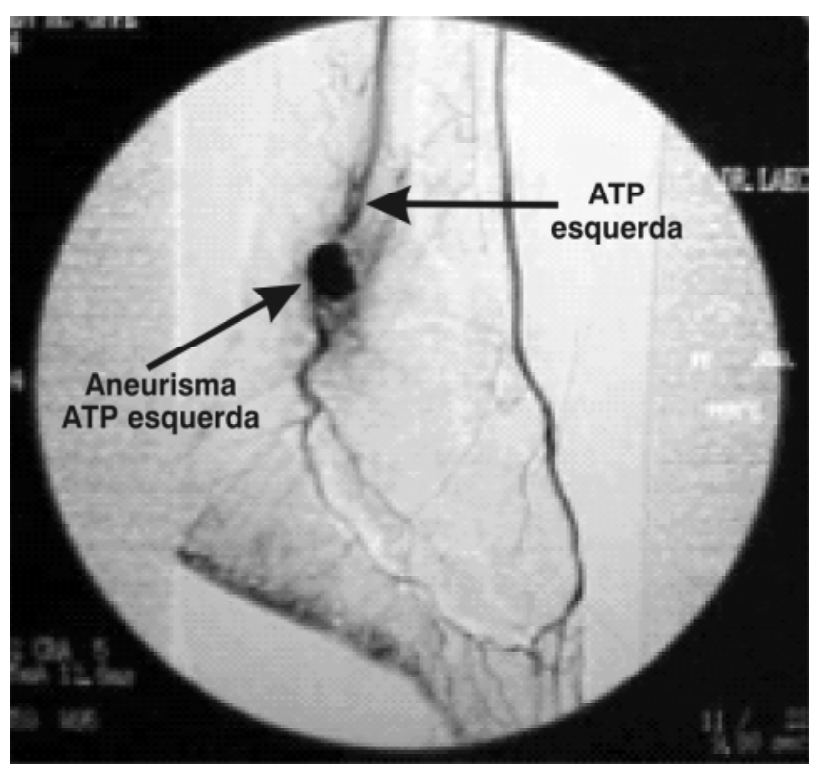

Figura 3 - Arteriografia, realizada no segundo internamento, demonstrando aneurisma fusiforme de ATP esquerda e a não-visualização distal da artéria peroneira já descrita na arteriografia anterior

ATP $=$ artéria tibial posterior.

\section{D iscussão}

A importância do relato e da discussão desse caso é a sua raridade, já que, apesar de haver na literatura descrição da ocorrência do aneurisma verdadeiro da ATP unilateral, não foi encontrado outro relato de aneurisma bilateral verdadeiro desta artéria. 0 utro aspecto importantedestecaso éa ausência deanormal idade no nível da ATP esquerda quando realizado 0 diagnóstico do aneurisma à direita; assim como ocorre em muitos casos com o aneurisma da artéria poplítea, houve desenvolvimento posterior de um aneurisma na artéria contralateral. M erece também destaque, neste caso, a presença do aneurisma trombosado da artéria peroneira esquerda, porém, a presença de aneurismas em sítios diferentes é também observada em outros tipos de aneurismas periféricos $1,2,6-10$.

0 diagnóstico clínico e os métodos complementares de imagem empregados são similares aos utilizados na avaliação de aneurismas periféricos localizados em outros sítios. N este caso, foram utilizados o eco-D oppler e a arteriografia, pois estes eram os métodos disponíveis no nosso serviço. Em relação ao tratamento cirúrgico, foi escolhida a técnica de ligadura da ATP direita, já que o paciente não apresentava anormalidades nas outras artérias da perna direita, enquanto quea opção pela revascularização da AT P esquerda foi devida à presença do aneurisma trombosado da artéria fibular esquerda. Essas técnicas são as utilizadas pela maioria dos autores $1,2,9,10,13,14$.

A possibilidade de o paciente apresentar um aneurisma em outra artéria não está afastada, e, desta forma, eleseguesob acompanhamento ambulatorial semestral, para exame físico e realização de eco-Doppler das principais artérias ${ }^{1,2,15,16}$.

0 paciente não apresentava história pregressa de trauma ou infecção que pudesse justificar o aparecimento do aneurisma. T ambém não apresentava anormalidades das provas inflamatórias, diminuindo a possibilidade de uma vasculite inflamatória ser a causa do aneurisma. 0 examehistopatológico confirmou o diagnóstico de aneurisma verdadeiro provocado por degeneração aterosclerótica. A confirmação da etiologia do aneurisma através do estudo histológico está de acordo com a literatura. A aterosclerose como causa do aneurisma, nestecaso, era o mais provável, já que este processo patológico é a etiologia mais comum dos aneurismas arteriais periféricos ${ }^{1,2,6-10}$.

\section{Referências}

1. Brito CJ. Aneurismas arteriais periféricos. In: $M$ affei $F H$. $D$ oenças vasculares periféricas. $3^{a}$ ed. Rio de Janeiro: M edsi; 2002. p. 1149-67.

2. Brito CJ, Azevedo Jr. AC, SchulzeGC. Aneurismasperiféricos. In: Brito CJ. Cirurgia vascular. Rio de Janeiro: Revinter; 2002. p. 534-50. 
3. D arwin E. Aorta e artérias ilíacas. In: D arwin E. D oenças vasculares: abordagens multidisciplinares ao diagnóstico e tratamento. Rio de Janeiro: Dilivros; 1999. p. 207, 487.

4. G raham LM . Femoral and poplitea aneurysms. In: $\mathrm{H}$ ai movici $H$, editor. V ascular surgery. Philadelphia: W B Saunders C 0.; 2000. p. 1345-56.

5. Vieira GN, Luz N W. Aneurismas das artérias dos membros inferiores. In: Bonamigo TP, Burihan E, Cinelli Jr. M, von Ristow A. D oenças da aorta e seus ramos: diagnóstico e tratamento. São Paulo: BYK; 1991. p. 59-72.

6. M iranda F. Aneurisma da artéria poplítea. In: Bonamigo TP, von Ristow A. Aneurismas. Rio de Janeiro: Dilivros; 2000. p. 252-8.

7. Galindo C C, Lima CA, C ardoso JE, G alindo Filho G, Costa VS, Penha FM. Aneurisma aterosclerótico isolado da artéria femoral superficial - relato de caso. J Vasc Br. 2003;2:145-7.

8. O liveira RS, Ferreira D A, T erra Júnior JA, Lenza RM, Assis Filho AC, N unesW . Ruptura deaneurismadaartériapoplítea: relato de caso e revisão daliteratura dosúltimos50 anos. J V asc Br. 2005;4:105-10.

9. Kanaoka T, M atsuura $\mathrm{H}$. A true aneurysm of the posterior tibial artery: a case report. Ann Thorac Cardiovasc Surg. 2004;10:317-8.

10. M onig SP, W alter M , Sorgatz S, Erasmi H . T rueinfrapopliteal artery aneurysms: report of two cases. J V asc Surg. 1996;24: 276-8.

11. Lima IA, Lima M F, Bernardes M V. Aneurisma isolado de artéria femoral profunda: relato de caso e revisão da literatura. J V asc Br. 2003;2:333-8.
12. H uber T S, Back M R, Ballinger R J, et al. U tility of magnetic resonance arteriography for distallower extremity revascularization. J V asc Surg. 1997;26:415-23.

13. Kauffman $P, P$ uech-L eão $P$. T ratamento cirúrgico do aneurisma da artéria poplítea: 32 anos de experiência. J V asc Br. 2002; 1:5-14.

14. M ahmood A, Salaman R, Sintler M, Smith SR, Simms M H, V ohra RK. Surgery of popliteal artery aneurysms: a 12-year experience. J V asc Surg. 2003;37:586-93.

15. D iwan A, Sarkar R, Stanley J C, Zelenock G B, W akefield TW . Incidence of femoral and popliteal artery aneurysmsin patients with abdominal aortic aneurysms. J V asc Surg. 2000;31:863-9.

16. Stone PA, Armstrong PA, Bandyk DF, et al. The value of duplex surveillance after open and endovascular popliteal aneurysm repair. J V asc Surg. 2005;41:936-41.

\section{Correspondência:}

Rodrigo Augusto de Aguiar Guedes

Rua Leonardo Bezerra C aval cante, 59/202, Jaqueira

CEP 52060-030 - Recife, PE

Tel.: (81) 3268.2842 / (81) 9976.6996

E-mail: raaguedes@smartsat.com.br 